\title{
Let sleeping worms lie
}

Sleep is a highly conserved process, but its original function is not yet clear. Previous work showed that overexpression of lin-3 (an orthologue of epidermal growth factor (EGF)) induced an ALAneuron-dependent sleep-like state in Caenorhabditis elegans through the activation of the EGF receptor let-23, although the physiological importance of this quiescence was not clear. Now, two studies reveal that C. elegans displays a sleep-like state in response to heat stress. These papers identify some of the mechanisms that mediate this quiescent state and suggest that it is necessary for recovery from cellular stress.

In one of the new studies, Hill et al. showed that when worms were exposed to a $35^{\circ} \mathrm{C}$ heat shock for 30 minutes, they exhibited a complete suppression of feeding and locomotor behaviour during and after the heat exposure, and that the post-heatexposure quiescence was dependent on the ALA neuron. In the other study, Nelson et al. found that silencing the ALA neuron sped recovery from heat-shock-induced quiescence, whereas optogenetically depolarizing the ALA neuron suppressed feeding and locomotor behaviour, confirming that ALA neuron activity is important and sufficient for quiescence.

Nelson et al. next showed that the ALA neuron expresses $f l p$-13-encoded neuropeptides and secretes these in response to heat stress. Worms in which $f l p-13$ overexpression was induced fell quiescent for several hours, and worms with a mutation in $f l p-13$ showed reduced suppression of feeding and locomotor behaviour in response to optogenetic stimulation of ALA neurons. Furthermore, worms with the $f l p-13$ mutation were resistant to the quiescence-promoting effects of lin-3 overexpression. Hill et al. found that worms with mutations in genes involved in lin-3 signalling were faster to start feeding and moving after a heat shock than were wild-type worms (WTs). Together, these findings suggest that, in response to heat stress (or in worms that overexpress lin-3), lin-3 signalling promotes ALA neuron activation and the secretion of flp-13-encoded neuropeptides, which in turn induce quiescence.

Next, Hill et al. showed that exposure to hyperosmotic or ethanol solutions, severe cold or a bacterial toxin also induced ALA-neurondependent quiescence. In addition, they found that, in response to moresevere heat stress $\left(37^{\circ} \mathrm{C}\right.$ or $\left.40^{\circ} \mathrm{C}\right)$, the initial quiescent bout was followed by a second, longer-lasting period of ALA-neuron-dependent quiescence. Together, these findings prompted the authors to determine whether the sleep-like state enables worms to recover from cellular stress.

Hill et al. showed that, after a $37^{\circ} \mathrm{C}$ heat shock, ALA-neurondefective mutant worms exhibited a longer-lasting upregulation in the expression of heat-shock protein 16.2 (HSP-16.2) and HSP-4 - chaperone proteins that restore protein homeostasis in stressed cells - than did WTs. In addition, compared with WTs, chaperone-defective mutant worms showed an increased duration of quiescence following heat stress, suggesting that impaired recovery from disruptions in protein homeostasis can extend quiescence. Remarkably, ALA-neuron-defective mutants were less likely to survive after a $40^{\circ} \mathrm{C}$ heat stress than were WTs, but a mutation in egl-4 that leads to spontaneous bouts of inactivity improved survival in
ALA-neuron-defective animals, implying that recovery from heat shock is promoted when C. elegans worms are inactive.

Overall, these studies demonstrate that cellular stress can induce a sleep-like state in C. elegans via lin-3-signalling-induced secretion of flp-13-encoded neuropeptides from the ALA neuron. These findings also provide evidence that this sleep-like state in C. elegans is key in promoting efficient recovery from the disruption of protein homeostasis that is brought about by cellular stress.

Natasha Bray
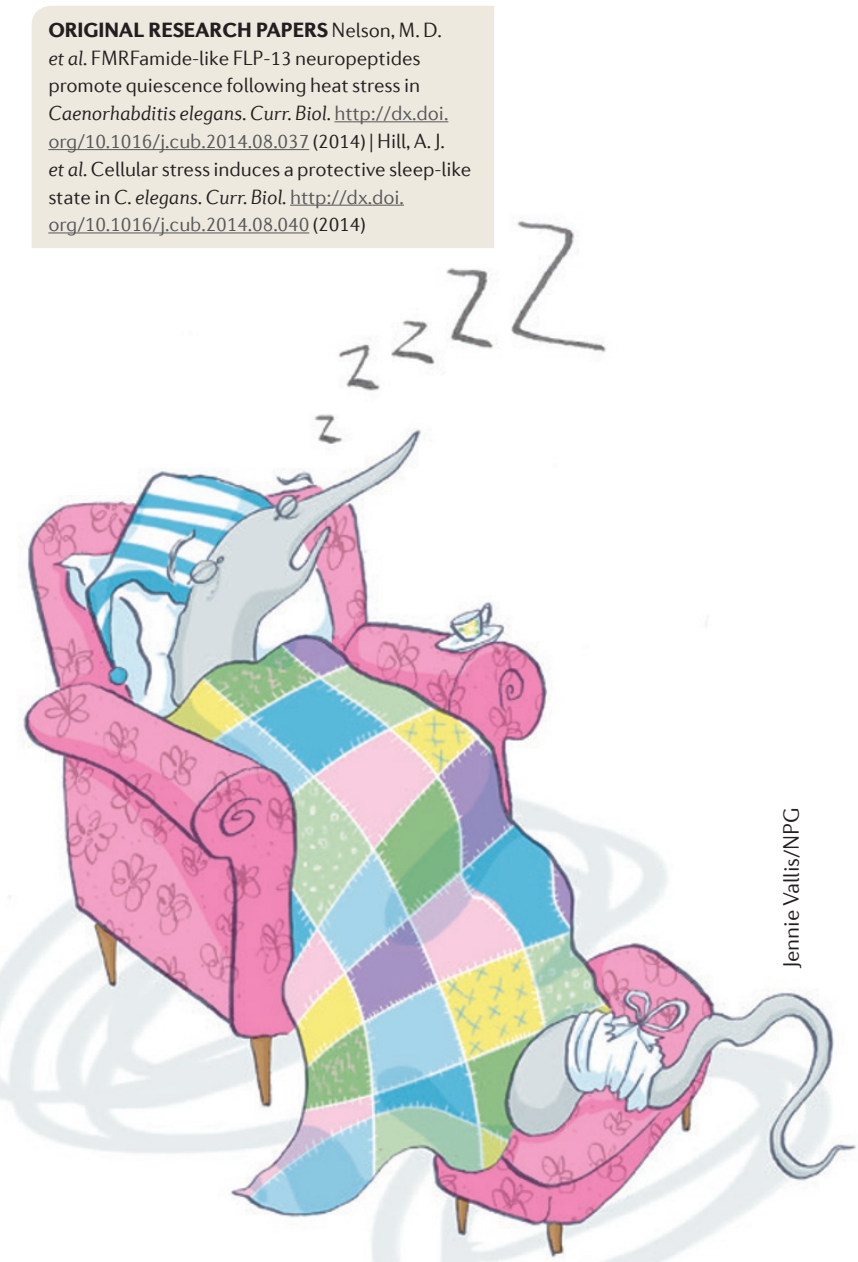